\title{
An Adaptive Network Model of the Role of the Gut-Brain Axis in Parkinson's Disease
}

\author{
Catherine GOETZINGER ${ }^{\mathrm{a}, \mathrm{b}}$, Korinzia TONIOLO ${ }^{\mathrm{c}, 1}$ and Jan TREUR ${ }^{\mathrm{d}}$ \\ ${ }^{a}$ Deep Digital Phenotyping Research Unit, Department of Population Health, \\ Luxembourg Institute of Health, Strassen, Luxembourg \\ ${ }^{b}$ University of Luxembourg, Faculty of Science, Technology and Medicine, \\ Luxembourg \\ ${ }^{c}$ Department of Management, University of Bologna, Italy \\ ${ }^{d}$ Social AI Group, Vrije Universiteit Amsterdam
}

\begin{abstract}
This paper presents an adaptive network model of the brain-gut axis and its related mechanisms that play major roles in the development of Parkinson's Disease. Simulations gave useful insight into how the biological and mental pathways interact with each other. In addition, the model provides information on the different time spans that are taken into consideration within these processes.
\end{abstract}

Keywords. Brain-Gut axis, Parkinson's Disease, adaptive network model, selfmodeling network.

\section{Introduction}

Parkinson's Disease (PD) is a systemic disease clinically defined by the degeneration of dopaminergic neurons in the brain. It has become the second most widespread neurodegenerative disorder in the world, expected to affect more than 10 million people worldwide by $2030[1,2]$. $\alpha$-synucleinopathy and motor impairment are two of the main signals of PD pathogenesis. They occur when $55 \%$ of the dopaminergic neurons are damaged in the substantia nigra, the area of the midbrain containing the nerve cells producing dopamine. Besides motor impairment, over time PD patients can show metabolic imbalance, with half of them experiencing constipation before the outset of other clinical features [3]. Recently, some studies have begun to explore the mechanisms that connect the gut and the brain. For example, changes in the composition of intestinal microbiota have been associated with neurological and neurodevelopmental disorders [4].

In this paper, we will specifically focus on the influence of gut microbiota mechanisms on PD mechanisms, analyzing computationally how such processes take place. This computational analysis addressing the different processes at different time scales involved, was performed based on multi-order adaptive networks modeled by the concept of self-modeling network.

\footnotetext{
${ }^{1}$ Corresponding Author: Korinzia TONIOLO, Department of Management, University of Bologna, Italy; Email: korinzia.toniolo2@unibo.it.
} 


\section{Background Literature}

The microbiota is composed by trillions of microorganisms living within our bodies. In the past decade, researchers have started to study it as one of the key regulators of the gut-brain axis, recognizing its important role for the central nervous system (CNS) [5]. The gut-brain axis is a bidirectional interaction, which integrates the cognitive and emotional centers of the brain with peripheral gastrointestinal mechanisms [3]. Recent studies on the gut-brain axis have revealed multiple ways the gut microbiota composition can affect people's daily lives and disease outcomes. Multiple factors can affect the microbiota composition over time, including infections, antibiotic medications, environmental stressors, and host genetics [6]. For example, it has been found that the microbiota of a PD patient tends to be characterized by reduced carbohydrate fermentation and butyrate synthesis capacity, increased proteolytic fermentation and production of deleterious amino acid metabolites, including $\mathrm{p}$-cresol and phenylacetylglutamine [7]. Further, PD patients' microbiota shows reduced levels of fecal short-chain fatty acids that may generate alteration in the enteric nervous system, causing constipation in PD patients [8]. Evidence from laboratories' investigation support the hypothesis of the connection between the complexity of gut microorganisms and PD pathogenesis [9-11].

Figure 1 provides an overview of how the connection between gut and brain mechanisms may occur in PD pathogenesis.

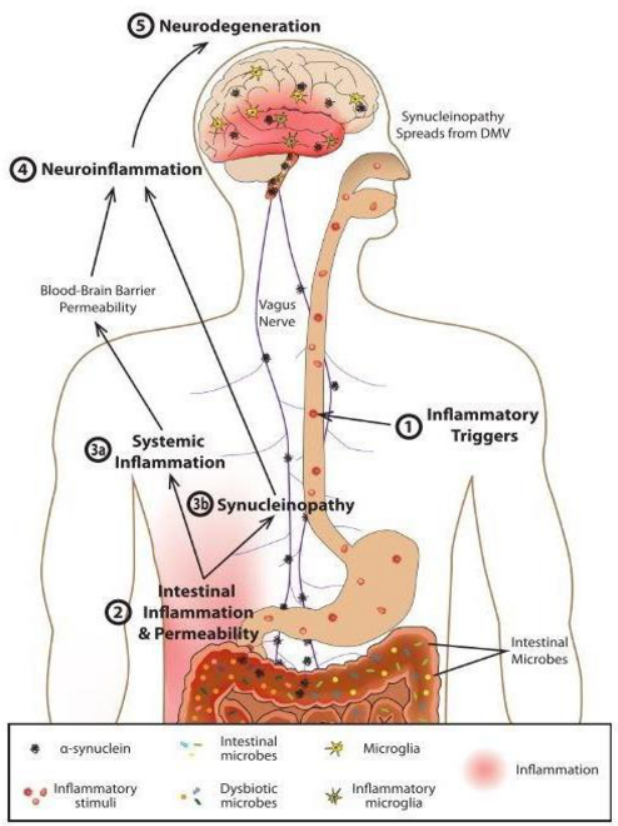

Figure 1. Model of gut-originating, inflammation-driven PD pathogenesis adopted from (4).

An inflammatory trigger, for example, a toxin or the intake of antibiotics, can initiate an inflammatory response in the gut and contribute to developing bad gut microbiota. The gut microbial toxins may then trigger the production of an increased aggregation of $\alpha$-synuclein ( $\alpha$-syn) protein in the enteric nervous system (ENS), which can be transmitted to the central nervous system $(\mathrm{CNS})$ through the vagus nerve. The presence 
of high aggregations of the $\alpha$-synuclein protein (synucleinopathy) in the gut is the main manifestation of PD. Peripheral inflammation can then trigger a system inflammatory response, increasing the likelihood of neuroinflammation and accelerating the pace of neurodegeneration.

In this context, some studies have started to investigate the use of probiotics for Parkison's disease prevention or treatment [12]. The presence of probiotic bacteria favors a healthy gut environment by modulating the host immune response and balancing the intestinal microflora.

Nutraceutical interventions aim to normalize the gut microbiome dysbiosis and improve biological outcomes in PD conditions. In this sense, regular intakes of probiotics can positively affect the gut microflora by increasing the presence of beneficial microorganisms [13]. Thus, the use of probiotics together with healthy diet habits can be seen as an adjuvant therapy to the use of standard PD medications, such as Levodopa. Brain cells in the substantia nigra within PD patients no longer produce the chemical dopamine, essential for brain neurons to communicate and control movement [14]. The chemical imbalance in PD causes physical symptoms such as resting tremors, stiffness, and slowness of movements, among others. Levodopa, a central nervous system agent, helps people with PD by replacing the chemical messenger dopamine [15]. It helps to alleviate the symptoms of PD by providing a supply of dopamine. It has to be noted, however, that Levodopa does not slow or reduce the progression of PD. Levodopa is an amino acid that is processed to dopamine in the brain. Today, Levodopa is the most effective drug used by PD patients [14].

\section{Network-Oriented Modeling}

To describe and analyze the influence of gut microbiota mechanisms on PD mechanisms computationally, we used a network-oriented modeling approach as described in $[16,17]$.

The introduced network model will model both the dynamics of the interaction processes within networks and of network evolution. Within PD we have processes that have very different time spans; some of the processes do occur on a daily basis (e.g., intake of fiber levels), whereas others only occur over the years (e.g., neurodegeneration). This type of model is declarative and characterized by connectivity characteristics, aggregation characteristics, and timing characteristics [17]:

- Connectivity characteristics: connections from states $X$ to $Y$, having connection weights $\omega_{X, Y}$ specifying their strengths

- Aggregation characteristics: each state $Y$ has a combination function $\mathbf{c}_{Y}$ that specifies how impact from all incoming connections on $Y$ is aggregated. Based on a list of basic combination functions bcf $_{i}$ (each with some parameters) provided by an available library, such a combination function can be specified by weights $\gamma_{i}$ and parameters $\boldsymbol{\pi}_{i, j}$ for these basic combination functions $\mathbf{b c f}_{i}$

- Timing characteristics: each state $Y$ has a speed factor $\eta_{Y}$ specifying how fast $Y$ changes

Furthermore, these models can be conceptualized in the form of matrices or labeled graphs. These conceptual graphs illustrate states and their connections between them plus some labels. States have varying activation levels over time. Moreover, the concept of weights of a connection is used for the strengths of impact of one state on another one. 
Finally, combination functions are used that represent the aggregation of multiple causal impacts on a state, and speed factors to represent the speed of change of a state, thus modeling the timing of the process. Next to matrices and labeled graphs, models can be formalized in a numeric format:

$$
Y(t+\Delta t)=Y(t)+\eta_{Y}\left[\mathbf{c}_{Y}\left(\omega_{X_{1}, Y} X_{1}(t), \ldots, \omega_{X_{k}, Y} X_{k}(t)\right)-Y(t)\right] \Delta t
$$

Eq. (1) represents how the states $X_{1}$ to $X_{k}$ from which $Y$ gets incoming connections affects the activation level of $Y$. The effect of the combination function $\mathbf{c}_{Y}(.$.$) on Y$ is exerted gradually over time, depending on speed factor $\eta_{Y}$. Eq. (1) are hidden in the software environment that can be used for simulation and analysis; see [17]. Within the above-mentioned software, more than 45 basic combination functions are included. The combination functions used in the present paper are presented in Table 1.

Table 1. The basic combination functions from the library used in the presented model.

\begin{tabular}{llll}
\hline & Notation & Formula & Parameters \\
\hline $\begin{array}{l}\text { Stepmod } \\
\text { stepmod }, \delta(V)\end{array}$ & 0 if $t \bmod \rho \leq \delta$, else 1 & $\rho$ repetition interval length, $\delta$ step time \\
$\begin{array}{l}\text { Advanced } \\
\text { logistic }\end{array}$ & alogistic $_{\sigma, \tau}\left(V_{1}, \ldots, V_{k}\right)$ & {$\left[\frac{1}{\left.1+\mathrm{e}^{-\sigma\left(V_{1}+\cdots+V_{k}-\tau\right.}\right)}-\right.$} & Steepness $\sigma>0$ \\
sum & $\left.\frac{1}{\left.1+\mathrm{e}^{\sigma \tau}\right)}\right]\left(1+\mathrm{e}^{-\sigma \tau}\right)$ & Excitability threshold $\tau$ \\
\hline
\end{tabular}

\section{Self-Modeling Networks for Adaptivity}

'Network characteristics' and 'network states' are by definition two distinct concepts for a network. Self-modeling networks are based on a self-model concept for part of their own network structure. A self-model represents certain network structure characteristics (e.g., connection weights or excitability thresholds) by network states. Hence self-models extend the states from the base level by additional states at a higher level, enabling adaptation of the network structure. This step is also called network reification. It is possible to create higher-order self-modeling levels, where network characteristics from one level are related to states from another level: second-order or higher-order adaptive networks [17].

In our scenario, self-modeling is added for those network characteristics that experience a change over time. Therefore, we end up with different levels of the model that interact with each other; a network model for the base network and its withinnetwork dynamics and a numerical model for the adaptivity of (some of) the network structure characteristics of the base network [17]. Self-modeling uses a network-oriented conceptualization similar to what was described above.

- Connectivity self-model

Self-model states $\mathbf{W}_{X, Y}$ are used to represent connectivity characteristics, i.e., connection weights $\omega_{X, Y}$

- Aggregation self-model

Self-model states $\mathbf{C}_{j, Y}$ are used to represent the first type of aggregation characteristics: combination function weights $\boldsymbol{\gamma}_{j, Y}$. Self-model states $\mathbf{P}_{i, j, Y}$ are used to represent the second type of aggregation characteristics: combination function parameters $\pi_{i, j, Y}$.

- Timing self-model 
Self-model states $\mathbf{H}_{Y}$ are used to represent timing characteristics: speed factors $\eta_{Y}$

In certain cases, the names using the letters $\mathbf{W}, \mathbf{C}, \mathbf{P}$ and $\mathbf{H}$ can be chosen in a different, more specific manner for example, $\mathbf{T}$ for excitability threshold parameter $\tau$.

The self-modeling construction can be iterated so that second-order (or even higherorder) adaptation can be modeled. An example of a second-order self-model state is a state $\mathbf{H w}_{X, Y}$ that represents the speed factor (adaptation rate) $\boldsymbol{\eta}$ of (first-order) self-model state $\mathbf{W}_{X, Y}$. This type of self-model states can be used in an adaptive network model to control the adaptation: decreasing activation values of $\mathbf{H w}_{X, Y}$ model make the adaptation slow down, and a value 0 causes a complete freezing of the adaptation.

\section{An integrative Adaptive Network Model}

Moving from the general premises, we first developed the conceptual model presented in Figure 2, which shows the connections between the gut mechanisms (on the left side of the graph) and the brain mechanisms (on the right side of the graph) which processes play a major role in PD pathogenesis. The blue boxes in Figure 2 represent two selfmodel states $\mathbf{W}_{X, Y}$ and one self-model state $\mathbf{T}_{Y}$.

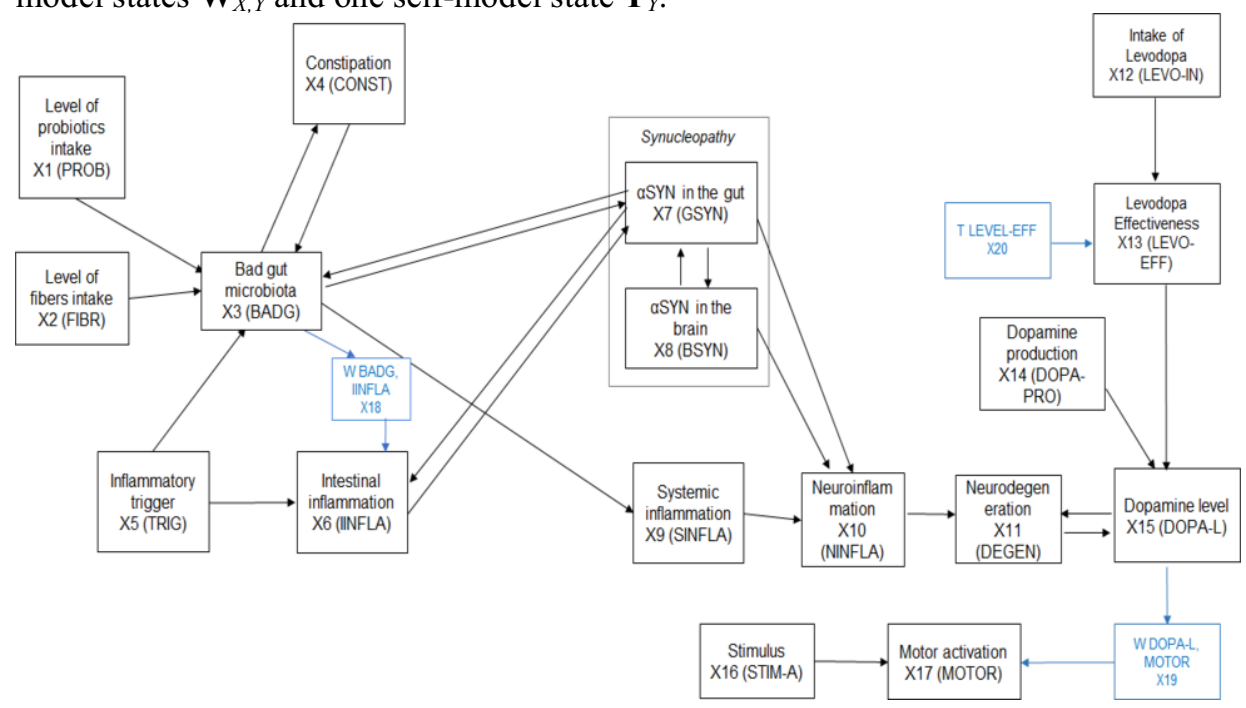

Figure 2 - Conceptual Model for the brain-gut axis in PD.

The designed self-modeling network model integrates both biological (physiological) and mental (neural) pathways. Both pathways interact in the so-called brain-gut axis which is represented at the base level (the pink plane in Figure 3). In PD, processes occur at different time spans and take a different amount of time to impact other processes. Therefore, an important addition to this model contributed here is an adaptive form of timing. Figure 3 represents a graphical 3D format that shows the base model on the bottom, and the self-model states are depicted at the next levels (reification levels). 


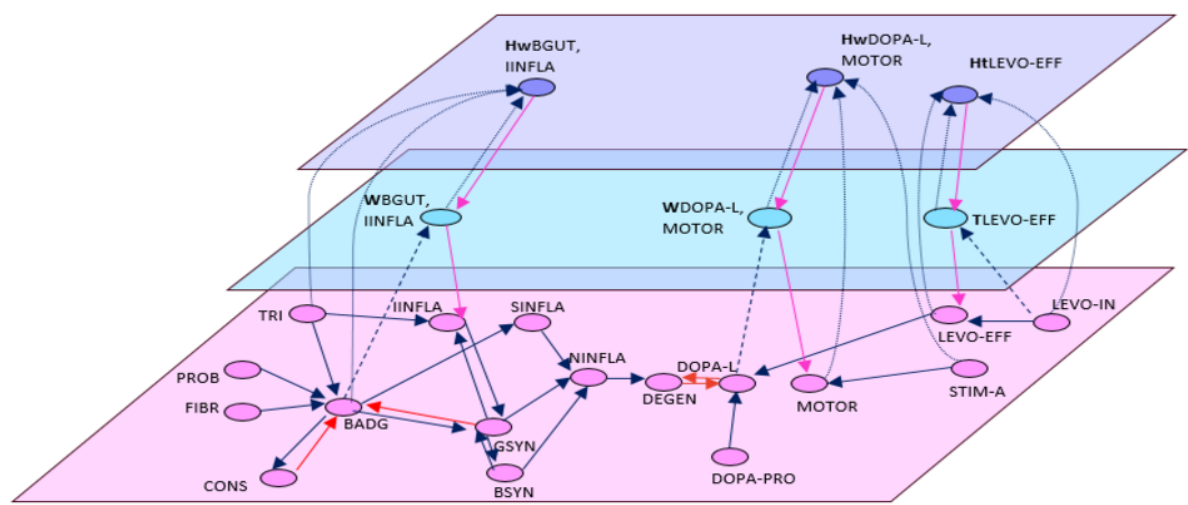

Figure 3. 3D figure of the connectivity of an integrative adaptive network model for the gut-brain axis in PD.

The states are labeled and described in Table 2. On the base level (pink plane) 'PROB', 'FIBR', 'TRI', 'DOPA-PRO' and 'LEVO-IN' are independent states that have no incoming influences yet influence other states. These independent states are timedependent and occur occasionally; therefore, the stepmod combination function was used for these states to generate repetitive patterns. For all the other states, we used the alogistic combination function.

On the base level, some arrows are red, meaning that they have a negative influence on the respective state. Through literature review, we found that constipation and $\alpha$-syn in the gut have a negative influence on the gut microbiota. Furthermore, we noted that neurodegeneration and dopamine levels negatively influence each other. Meaning that low dopamine levels negatively affect neurodegeneration, and with increased neurodegeneration, it has a negative impact on dopamine levels.

Reading this $3 \mathrm{D}$ figure, we can see that probiotic $\left(\mathrm{X}_{1}\right)$ and fiber $\left(\mathrm{X}_{2}\right)$ intake influence the gut microbiota. If you have low levels of both, you tend to develop a bad gut microbiota $\left(\mathrm{X}_{3}\right)$. In addition, inflammatory triggers $\left(\mathrm{X}_{5}\right)$ such as toxins influence the bad gut composition. Having a bad gut composition creates constipation $\left(\mathrm{X}_{4}\right)$, which in return negatively affects the bad gut composition. Moreover, the bad gut composition has an influence on the system inflammation $\left(\mathrm{X}_{9}\right)$ as well as on the $\alpha$-syn in the gut $\left(\mathrm{X}_{7}\right)$.

The synucleinopathy $\left(\mathrm{X}_{7}\right.$ and $\left.\mathrm{X}_{8}\right)$ and the intestinal inflammation $\left(\mathrm{X}_{6}\right)$ affect each other. In addition, synucleinopathy influences neuro-inflammation $\left(\mathrm{X}_{10}\right)$, which means that neurodegeneration $\left(\mathrm{X}_{11}\right)$ is triggered. In addition, neurodegeneration increases by low levels of dopamine chemicals $\left(\mathrm{X}_{15}\right)$. The latter is affected by the production of dopamine $\left(\mathrm{X}_{14}\right)$ in the brain.

As stated in the background section, Levodopa is the most common drug used in PD. It helps to increase the level of dopamine in the brain. Therefore, levodopa intake $\left(\mathrm{X}_{12}\right)$ and its efficacy $\left(\mathrm{X}_{13}\right)$ affect dopamine levels. On an everyday basis, individuals receive stimulus for activation $\left(\mathrm{X}_{16}\right)$, which by consequences, therefore, triggers your motor activation $\left(\mathrm{X}_{17}\right)$.

As already mentioned in the previous section, the present models depicting the gutbrain axis in PD deals with states occurring at various time scales. Intake of inflammatory triggers can happen on a regular occasion, whereas the bad gut microbiota composition happens throughout a longer period. In order to adjust for such dynamics, the model includes time regulations. In the 3D figure, on the first adaptive level (blue plan), a weight connection was added to affect the inflammation trigger connection on intestinal 
inflammation $\left(\mathrm{X}_{6}\right)$. This is done by adding state $\mathbf{W}_{\text {BADG,IINFLA }}\left(1^{\text {st }}\right.$ order self-model level $)$ into our model. Bad gut composition makes you more sensible for intestinal inflammation and thus strengthens the link between the inflammatory trigger and intestinal inflammation. Therefore, $\mathbf{W}_{\mathrm{BADG}, \text { IINFLA }}\left(\mathrm{X}_{18}\right)$ will regulate the impact on how much inflammatory triggers influence intestinal inflammation. Another weight connection on this first adaptation level was created between state $\mathrm{X}_{12}$ and $\mathrm{X}_{17}, \mathbf{W}_{\text {DOPA- }}$ L,MOTOR $\left(\mathrm{X}_{19}\right)$. The dopamine level makes you more sensible for motor activation. The added $\mathbf{W}_{\text {DOPA-L,MOTOR }}$ state, therefore, strengths the link between the stimulus for activation state and motor activation state. Over time, the effectiveness of Levodopa decreases even, if the intake of the drug increases. In order to adjust for this dynamic process in the current model, an adaptive excitability threshold $\mathbf{T}_{\text {LEVEL-EFF }}\left(\mathrm{X}_{20}\right)$ was added to weaken the effectiveness of Levodopa over time.

On the second level of the model, the $\mathbf{H}$-states were added as a form of speed control for adaptation. Therefore $\mathbf{H w}_{\text {BADG,IINFLA }}\left(\mathrm{X}_{21}\right)$ will regulate the learning speed of $\mathbf{W}_{\text {BADG,IINFLA }}$. Similarly, $\mathbf{H}_{\text {DOPA-L, MOTOR }}\left(\mathrm{X}_{22}\right)$ will regulate the learning speed of $\mathbf{W}_{\text {DOPA- }}$ L, MOTOR and $\mathbf{H}_{\mathbf{T}_{\text {LEVEL-EFF }}}\left(\mathrm{X}_{23}\right)$ will regulate the learning speed of $\mathbf{T}_{\text {LEVEL-EFF, }}$ respectively.

Table 2. State labels and description

\begin{tabular}{|c|c|c|}
\hline States & Label & Description \\
\hline $\mathrm{X}_{1}$ & PROB & Probiotics intake \\
\hline $\mathrm{X}_{2}$ & FIBR & Fibers intake \\
\hline $\mathrm{X}_{3}$ & BADG & Bad gut microbiota \\
\hline $\mathrm{X}_{4}$ & CONST & Constipation \\
\hline $\mathrm{X}_{5}$ & TRIG & Inflammatory trigger \\
\hline $\mathrm{X}_{6}$ & IINFLA & Intestinal inflammation \\
\hline $\mathrm{X}_{7}$ & GSYN & $\alpha$-syn protein in the Gut \\
\hline $\mathrm{X}_{8}$ & BSYN & $\alpha$-syn protein in the Brain \\
\hline $\mathrm{X}_{9}$ & SINFLA & Systemic inflammation \\
\hline $\mathrm{X}_{10}$ & NINFLA & Neuroinflammation \\
\hline$X_{11}$ & DEGEN & Neurodegeneration \\
\hline $\mathrm{X}_{12}$ & LEVO-IN & Levodopa intake \\
\hline $\mathrm{X}_{13}$ & LEVO-EFF & Levodopa effectiveness \\
\hline $\mathrm{X}_{14}$ & DOPA-PRO & Dopamine production \\
\hline $\mathrm{X}_{15}$ & DOPA-L & Dopamine level \\
\hline $\mathrm{X}_{16}$ & STIM-A & Stimulus for activation \\
\hline $\mathrm{X}_{17}$ & MOTOR & Motor activation \\
\hline $\mathrm{X}_{18}$ & $\mathbf{W}_{\mathrm{BADG}, \text { IINFLA }}$ & $\begin{array}{l}\text { Self-model state for the weight of the connection from BADG to } \\
\text { IINFL }\end{array}$ \\
\hline $\mathrm{X}_{19}$ & $\mathbf{W}_{\text {DOPA-L, MOTOR }}$ & $\begin{array}{l}\text { Self-model state for the weight of the connection from DOPA-L to } \\
\text { MOTOR }\end{array}$ \\
\hline$X_{20}$ & $\mathbf{T}_{\text {LEVEL-EFF }}$ & Self-model state for the excitability threshold of LEVEL-EFF \\
\hline $\mathrm{X}_{21}$ & $\mathbf{H}_{\mathbf{W}_{\text {BADG, IINFLA }}}$ & Self-model state for adaptation speed of $\mathbf{W}_{\text {BADG, IINFLA }}$ \\
\hline $\mathrm{X}_{22}$ & $\mathbf{H}_{\mathbf{W}_{\text {DOPA-L, MOTOR }}}$ & Self-model state for adaptation speed of $\mathbf{W}_{\text {DOPA-L, мOTOR }}$ \\
\hline $\mathrm{X}_{23}$ & $\mathbf{H}_{\text {TLEVEL-EFF }}$ & Self-model state for adaptation speed of $\mathbf{T}_{\text {LEVEL-EFF }}$ \\
\hline
\end{tabular}

\section{Simulation of the Integrative Adaptive Network Model}

Within the present section, we will discuss the findings of the simulation that was generated using the software environment, and the specification by role matrices, detailing the network characteristics, which are presented in the Appendix available as Linked Data at URL https://www.researchgate.net/publication/353764251. Figure 4 shows the simulation representing the brain-gut axis process in PD. This scenario shows 
a adaptation effect after the inflammatory trigger intake, the stimulus activation, and the Levodopa effectiveness.

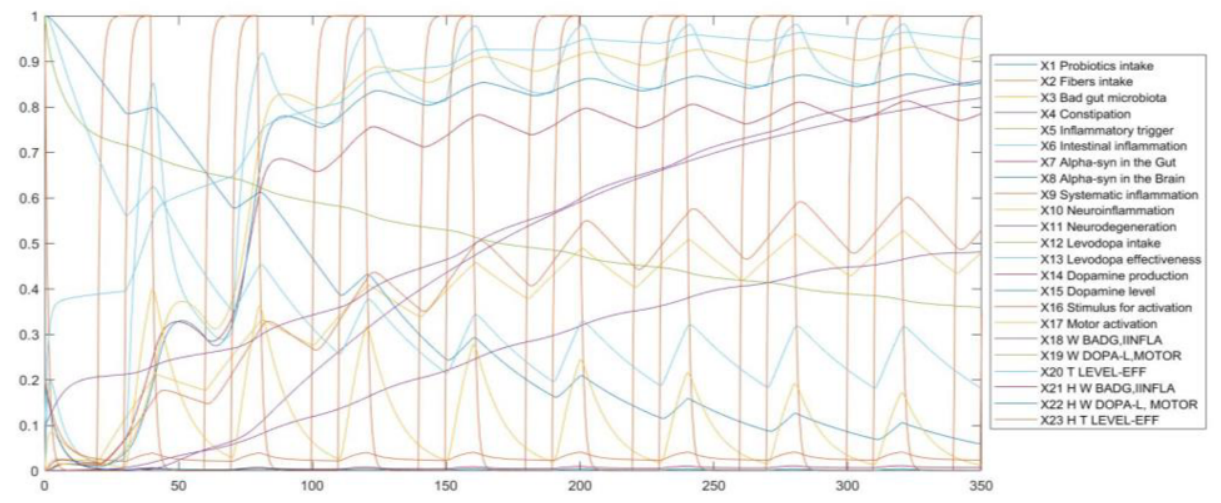

Figure 4. Simulation of the adaptive network model.

The simulation shows that probiotics and fiber levels continuously decrease over time due to an increasingly bad gut composition. With this, constipation slowly worsens. States $\mathrm{X}_{21}, \mathrm{X}_{22}, \mathrm{X}_{23}$, the adaptive connection weights and excitability threshold are very slow in the present model as can be spotted at the bottom of the simulation. Due to a slow adaptation rate of the model ( $\left.\mathbf{W}_{\mathrm{BADG}, I I N F L A}\right)$, intestinal inflammation only expresses after a certain amount of time once the influence of the inflammatory trigger is high enough to affect the intestinal inflammation. The same holds for the Motor activation that slowly decreases over the time span $\left(\mathbf{W}_{\text {DOPA-L, MOTOR }}\right)$. The excitability threshold $\left(\mathbf{T}_{\text {LEVEL-EFF }}\right)$ is slow at the beginning of the simulation and increases after 100-time points. Therefore, the Levodopa effectiveness remains high at the beginning of the simulation at thus decreases with increasing $\mathbf{T}_{\text {LEVEL-EFF. }}$

\section{Discussion}

The presented model and its respective simulation is the first computational representation of the brain-gut axis processes and mechanisms that play major roles in PD. This simulation is useful because it gives insight into how the biological and mental pathways interact with each other. In addition, the model provides information on the different time spans that are taken into consideration within these PD processes.

Nevertheless, the present model remains a simplified version of the PD pathogenesis and further work in computational modeling could consider, for example, the impact of the mitochondria process in PD. Furthermore, future studies may have a closer look at specific probiotics and their influence on the brain-gut axis in PD. These could eventually be helpful in the prevention strategies for PD. 


\section{References}

[1] Burgueño JF, Abreu MT. Epithelial Toll-like receptors and their role in gut homeostasis and disease. Nat Rev. Gastroenterol Hepatol. 2020 May;17(5):263-78.

[2] Marras C, Beck JC, Bower JH, Roberts E, Ritz B, Ross GW, et al. Prevalence of Parkinson's disease across North America. npj Parkinson's Disease. 2018 Jul 10;4(1):1-7.

[3] Dogra N, Mani RJ, Katare DP. The gut-brain axis: two ways signaling in Parkinson's disease. Cell Mol Neurobiol [Internet]. 2021 Mar 1 [cited 2021 Sep 14]; Available from: https://doi.org/10.1007/s10571 021-01066-7

[4] Houser MC, Tansey MG. The gut-brain axis: is intestinal inflammation a silent driver of Parkinson's disease pathogenesis? npj Parkinson's Disease. 2017 Dec;3(1):3.

[5] Wang Y, Kasper LH. The role of microbiome in central nervous system disorders. Brain Behav Immun. 2014 May; 38:1-12.

[6] Cryan JF, O'Riordan KJ, Cowan CSM, Sandhu KV, Bastiaanssen TFS, Boehme M, et al. The microbiotagut-brain axis. Physiological Reviews. 2019 Oct 1;99(4):1877-2013.

[7] Cirstea MS, Yu AC, Golz E, Sundvick K, Kliger D, Radisavljevic N, et al. Microbiota composition and metabolism are associated with gut function in Parkinson's disease. Mov Disord. 2020 Jul;35(7):120817.

[8] Koh A, De Vadder F, Kovatcheva-Datchary P, Bäckhed F. From dietary fiber to host physiology: shortchain fatty acids as key bacterial metabolites. Cell. 2016 Jun 2;165(6):1332-45.

[9] Bedarf JR, Hildebrand F, Coelho LP, Sunagawa S, Bahram M, Goeser F, et al. Functional implications of microbial and viral gut metagenome changes in early stage L-DOPA-naïve Parkinson's disease patients. Genome Medicine. 2017 Apr 28;9(1):39.

[10] Hill-Burns EM, Debelius JW, Morton JT, Wissemann WT, Lewis MR, Wallen ZD, et al. Parkinson's disease and Parkinson's disease medications have distinct signatures of the gut microbiome. Mov Disord. 2017 May;32(5):739-49.

[11] Keshavarzian A, Green SJ, Engen PA, Voigt RM, Naqib A, Forsyth CB, et al. Colonic bacterial composition in Parkinson's disease. Mov Disord. 2015 Sep;30(10):1351-60.

[12] Castelli V, d'Angelo M, Quintiliani M, Benedetti E, Cifone MG, Cimini A. The emerging role of probiotics in neurodegenerative diseases: new hope for Parkinson's disease? Neural Regen Res. 2021 Apr;16(4):628-34.

[13] Wong CB, Kobayashi Y, Xiao J. Probiotics for preventing cognitive impairment in Alzheimer's disease [Internet]. Gut Microbiota - Brain Axis. IntechOpen; 2018 [cited 2021 Sep 14]. Available from: https://www.intechopen.com/chapters/62125

[14] Gandhi KR, Saadabadi A. Levodopa (L-Dopa). In: StatPearls [Internet]. Treasure Island (FL): StatPearls Publishing; 2021 [cited 2021 Sep 14]. Available from: http://www.ncbi.nlm.nih.gov/books/NBK482140/

[15] Lewitt PA. Levodopa for the treatment of Parkinson's disease. N Engl J Med. 2008 Dec 4;359(23):246876.

[16] Treur J. Network-oriented modeling: addressing complexity of cognitive, affective and social interactions [Internet]. Springer International Publishing; 2016 [cited 2021 Sep 14]. (Understanding Complex Systems). Available from: https://www.springer.com/gp/book/9783319452111

[17] Treur J. Network-oriented modeling for adaptive networks: designing higher-order adaptive biological, mental and social network models [Internet]. Springer International Publishing; 2020 [cited 2021 Sep 14]. (Studies in Systems, Decision and Control). Available from: https://www.springer.com/gp/book/9783030314446 